\section{KOMPASS}

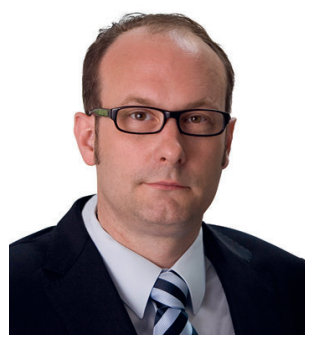

Volker Harth

Zentralinstitut für Arbeitsmedizin und Maritime Medizin (ZfAM), Universitätsklinikum Hamburg-Eppendorf (UKE), Hamburg, Deutschland

\title{
Umweltbedingte Lungen- erkrankungen diagnostizieren und therapieren - aktuelle Themen aus der Arbeits- und Umweltmedizin
}

Sehr geehrte Kolleginnen und Kollegen, liebe Leserinnen und Leser,

akute respiratorische Erkrankungen und chronische Lungenerkrankungen (Lungenkrebs, chronisch-obstruktive Lungenerkrankung und Asthma bronchiale) sind sowohl in Deutschland als auch weltweit von hoher gesundheitsökonomischer und gesellschaftlicher Relevanz. Die Krankheitskosten dieser Entitäten werden allein in Deutschland durch das statistische Bundesamt auf über 16 Milliarden Euro pro Jahr geschätzt [1]. Gleichzeitig tragen sie zu einer hohen Krankheitslast und Sterblichkeit in der Bevölkerung bei. Weltweit sterben Schätzungen zufolge jedes Jahr allein mehr als 4 Millionen Menschen an COPD. Aufgrund dieser besonderen Relevanz werden Präventionsstrategien nicht nur von Seiten der Weltgesundheitsorganisation (WHO) konzipiert und unterstützt [2], sondern auch auf europäischer Ebene. Ein gelungenes Beispiel für eine konzertierte Aktion gibt der in dieser Ausgabe des KARGER KOMPASS PNEUMOLOGIE veröffentlichte Bericht über das europäische Treffen verschiedener Fachgesellschaften zu Prävention und Management chronischer Atemwegserkrankungen in Vilnius, Litauen, im März 2018 wieder [3]. Hier wurden multisektorale
Versorgungswege aufgezeigt und in der Abschlusserklärung (Vilnius Declaration) die Auswirkungen der Luftverschmutzungen auf die Gesundheit der Bevölkerung aufgezeigt.

\section{Leider übersehen wir in unserem} ärztlichen Praxisalltag allzu zu oft, dass

die Ursachen für Atemwegs- und

Lungenerkrankungen häufig direkt in der Arbeits- oder Umwelt des betroffenen

Patienten begründet liegen.

Über die gesundheitliche Bedeutung von Stickoxiden und Feinstäuben wurde gerade in Deutschland in den vergangenen Monaten im Kontext der Fahrverbote für Dieselfahrzeuge kontrovers und intensiv diskutiert. Lassen Sie mich an dieser Stelle daher einmal mehr auf die Relevanz arbeits- und umweltbedingter (Lungen-)Erkrankungen hinweisen. Leider übersehen wir in unserem ärztlichen Praxisalltag allzu oft, dass die Ursachen für Atemwegs- und Lungenerkrankungen häufig direkt in der Arbeits- oder Umwelt des betroffenen Patienten begründet liegen. Neben den akuten Belastungen, deren bronchiale Wirkung in ihrem zeitlichen Zusammenhang in der Regel leicht nachzuvollziehen ist, muss gerade den chronischen Einflüssen mit ihrer oftmals jahrzehntelangen Expositions- und Latenzzeit unsere besondere Aufmerksamkeit gelten. Insbesondere die nur eingeschränkt mögliche Erhebung lang zurückliegender arbeitsbedingter Expositionen erschweren es der Ärztin/dem Arzt, einen möglichen beruflichen Kausalzusammenhang herzustellen und die gesetzlich geforderte Verdachtsanzeige auf eine Berufskrankheit zu stellen. Die ärztliche Meldepflicht des Verdachts auf eine Berufskrankheit (BK) ist, ähnlich der Meldepflicht im Infektionsschutzgesetz, rechtlich verankert. Die ärztliche Anzeige ist aber nur Ausdruck eines Verdachts und muss nicht den Anspruch auf einen Vollbeweis erheben. Bei einem Unterlassen der Anzeige wird den betroffenen Patienten durch die Versäumnis einer BK-Meldung jedoch die Möglichkeit genommen, Maßnahmen zur Prävention bzw. Rehabilitation sowie eine ihrem Erkrankungsbild entsprechende finanzielle Entschädigung durch den Unfallversicherungsträger zu erhalten.

Einen besonderen Fall einer Verdachtsdiagnose auf eine Berufskrankheit stellen die Autoren Yabuuchi et al. in ihrer Kasuistik über eine Aluminose der Atemwege mit wahrscheinlich sekundärer pleuroparenchymaler Fibroelastose dar [4]. Dabei han-

\section{KARGER}

Fax +497614520714

information@karger.com

www.karger.com
(๑) 2019 S. Karger GmbH, Freiburg

Accessible online at: www.karger.com/kkp
Prof. Dr. Volker Harth

Zentralinstitut für Arbeitsmedizin und Maritime Medizin (ZfAM)

Universitätsklinikum Hamburg-Eppendorf (UKE)

Seewartenstraße 10, Haus 1, 20459 Hamburg, Deutschland

harth@uke.de 
delt es sich um ein klassisches Beispiel dafür, dass die Ableitung von Präventionsmaßnahmen am Arbeitsplatz zu spät erfolgte, so dass der Patient an den Folgen seiner aluminiumbedingten Fibroelastose viel zu früh verstorben ist.

Der Wissenstransfer von Kollege Dr. Lars Hagmeyer [5] zeigt eindrücklich, dass die E-Zigarette nicht bedenkenlos zur Raucherentwöhnung empfohlen werden sollte. Die proteomischen Analysen der Signalwege weisen darauf hin, dass die Basis-Trägersubstanz einen dem Zigarettenrauch ähnelnden modifizierenden Effekt auf zellulärer Ebene auslösen kann. Oberstes Ziel der Präventionsstrategie zur Raucherentwöhnung muss daher die Karenz jeglicher inhalativer Noxen bleiben.

In dieser Ausgabe ist einmal mehr gelungen, verschiedene Aspekte arbeits- und umweltbedingter Erkrankungen zu vereinen. Ich hoffe, diese Ausgabe findet Ihr Interesse, und wünsche Ihnen eine interessante und spannende Lektüre.

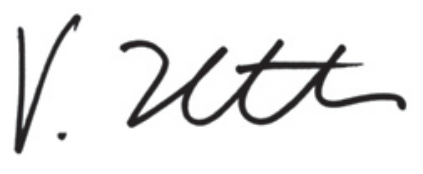

Ihr Professor Dr. Volker Harth

\section{Literatur}

1 Statistisches Bundesamt: Herz-Kreislauf-Erkrankungen verursachen die höchsten Kosten. Pressemitteilung Nr. 347 vom 29. September 2017, www.destatis.de/DE/Presse/Pressemitteilungen/2017/09/PD17_347_236.html (letzter Abruf: 12.06.2019).

2 World Health Association (WHO): Global Action Plan for the Prevention and Control of NCDs 2013-2020, WHO Press, Genf, 2013, https://apps.who.int/iris/bitstream/ handle/10665/94384/9789241506236_eng.pdf; jsessionid $=433351 C E 9 C 2035 E C 2 F 7728 A 7 E A$ 30A0D4? sequence $=1$ (letzter Abruf: 12.06.2019).

$\checkmark 3$ Valiulis AV, Bousquet J, Veryga A, et al.: Erklärung von Vilnius zu chronischen Atemwegserkrankungen: multisektorale Versorgungswege, die angeleitetes Selbstmanagement, mHealth und Luftverschmutzung bei der Versorgung chronischer Atemwegserkrankungen einschließen. Karger Kompass Pneumol 2019; 7:185-192.

4 Yabuuchi Y, Goto H, Nonaka M, et al.: Ein Fall von Aluminose der Atemwege mit wahrscheinlich sekundärer pleuroparenchymaler Fibroelastose. Karger Kompass Pneumol 2019;7:222-226.

5 Hagmeyer L: E-Zigarette hat weitreichende biologische Auswirkungen auf das Bronchialepithel - Erkenntnisse aus proteomischen Analysen. Karger Kompass Pneumol 2019;7:194-195. 